\title{
State of the Work Diagram of the Artificial Heart
}

\author{
G. Konieczny*, Z. Opilski, T. Pustelny and E. Maciak \\ Department of Optoelectronics, Silesian University of Technology \\ Krzywoustego 2, 44-100 Gliwice, Poland
}

\begin{abstract}
This paper presents the results of investigations on novel pressure measurement methods to be for used in the project "Polish Artificial Heart". The investigations concerned possible pressure sensors for noninvasive blood pressure measurements in selected points around the pneumatic ventricular assist device (POLVAD). The fiber pressure sensor and the possibility of pressure measurements with piezofoils were tested. The paper includes preliminary results and development plans.
\end{abstract}

PACS numbers: 42.79.-e, 42.81.-i, 07.07.Df, 47.63.Cb

\section{Introduction}

In the case of heart problems caused by diseases, mechanical support is one of the methods of helping heart muscle recovery [1-4]. The main purpose of the ventricular assist device (VAD) is to aid the human circulatory system by introducing an external blood pump connected with the human vascular system. This kind of support relieves the heart from a large part of its burden, thus allowing its faster recovery. The ventricular assist device (Fig. 1) is controlled by a pneumatic driving unit (PDU) of the POLPDU-401 type from the Polish Cardiac Assist System (POLCAS) [5, 6]. At the moment by using PDU the medical staff is able to set both the volume and the rate of blood pumped during one cycle. There is no feedback, so the driving unit provides no information about the current status of prosthesis.

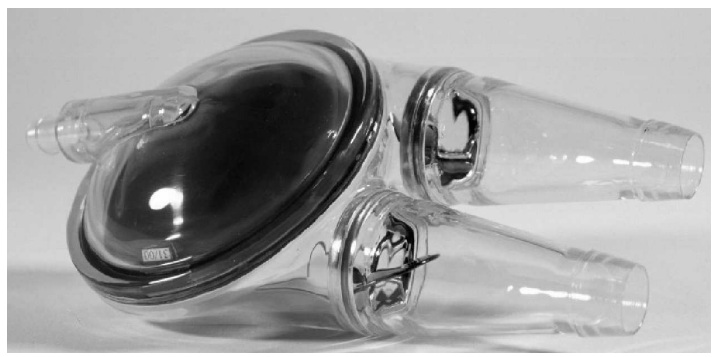

Fig. 1. Extracorporeal, pneumatic ventricular assist device POLVAD (FRK, Zabrze) [7].

Present efforts concern the application of the monitoring system for $\mathrm{VAD}$, that would supply information about the current state of the device (e.g. blood pressure, membrane position), and permit to monitor in the

\footnotetext{
* corresponding author; e-mail: grzegorz.konieczny@polsl.pl
}

real time of prosthesis and fully automatic operation in future. This paper presents recent researches concerning the blood pressure measurement system.

\section{Conducted researches}

The main purpose of the conducted researches, was to introduce the pressure measurement system for use in POLVAD prosthesis.

Project specifications required of the pressure sensor to meet certain specifications:

- blood pressure measurements without direct contact with the blood environment,

- measured pressure range $(-100$ to $+400 \mathrm{mmHg})$,

- possibility of measuring changes of $5 \mathrm{mmHg} / \mathrm{ms}$ dynamicity.

The research includes the results of testing two possible pressure sensing methods: the fiber pressure sensor and the sensor based on piezoelectric foils.

\subsection{Fiber pressure sensor}

The first investigated sensing device was a fiber pressure sensor manufactured by FISO C. The main advantages of using this fiber sensor are resistance to radio and electromagnetic interference, the possibility of sensing remote places and the size of the sensing element (a total fiber diameter is $\approx 1 \mathrm{~mm}$ ). A significant advantage to electrical sensors is the lack of additional wires (one fiber measurement system). The fiber is coated with a teflon layer which makes it bio compatible.

The measurement circuit consisted of a conditioner and a FOP-M fiber sensing element. For data acquisition and processing a special application, programmed with LabView 8.0, was used. The operation of the sensor 
bases on a Fabry-Perot interferometer, situated at the tip of the FOP-M fiber, which changes its dimensions when pressure is applied. For a given pressure, concrete wavelengths are amplified in the reflected spectrum. The conditioner is responsible for introducing light into the sensing fiber, detecting reflected light, and translating it into pressure changes expressed in required units [8].

The measured pressure range, guaranteed by the manufacturer, meets the project requirements. The sensor was tested for compliancy with other project directives.

In order to test the reproducibility of pressure measurements two FOP-M sensors were put in the same cavity (Fig. 2).

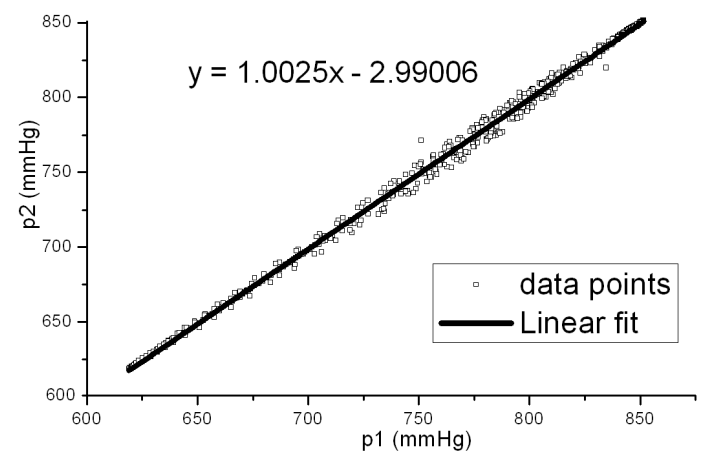

Fig. 2. Results of pressure measurements using two FOP-M sensors in the same environment.

The readings from both sensors are similar. Minor differences are below the required accuracy level and can be caused by uneven pressure inside the test cavity.

Since there was no information about dynamic pressure measurement capabilities of the sensor, such tests were carried out.

The outcome of this investigation has been shown in Fig. 3. The graph shows both the characteristics of the measured pressure and the dynamic pressure changes. The results indicate that the fiber sensor is capable of measuring dynamic pressure changes faster than $9 \mathrm{mmHg} / \mathrm{ms}$, which is enough to comply with project specifications $(5 \mathrm{mmHg} / \mathrm{ms})$.

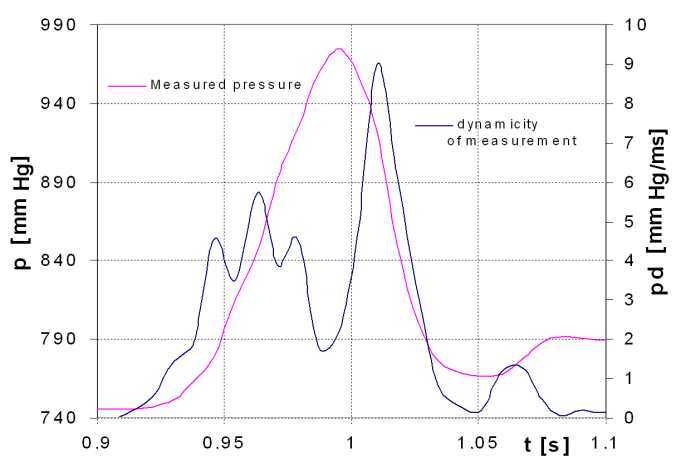

Fig. 3. Dynamic measurement tests of the fiber sensor.
The conducted research shows a wide potential application of fiber sensors in blood pressure measurements.

\subsection{Piezofoils}

Piezoelectric foils manufactured by Measurement Specialties@ were investigated as to their possible using in the pressure changes detection system. They require two wires, one extra wire compared with the fiber sensor.

Piezofoils act as a very low-efficiency voltage source. Pressure changes, among other factors (e.g. temperature and light intensity changes, movement), cause charge generation on the piezofoil and, therefore, inflict voltage changes at the sensors output terminals of the sensors. Due to the low efficiency of this "voltage source" it is crucial to use a differential amplifier with a low input bias current in order to acquire voltage changes, and to prevent a discharge of the sensing element during measurements. In the introduced testing circuit (Fig. 4), the INA116 instrumentation amplifier was used. Its input bias current is not greater than $2 \mathrm{fA}$.

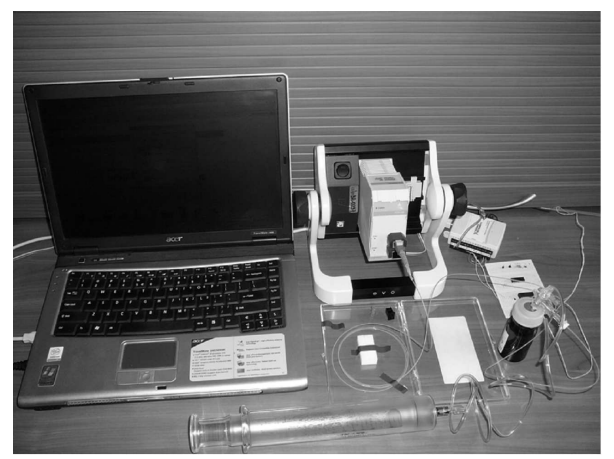

Fig. 4. Measurement equipment for piezoelectric foil tests.

As a reference sensor the FOP-M fiber sensor described in Sect. 2.1 was used. Both the piezofoil and the fiber sensor were placed in the same volume, where pressure changes were being induced.

The tests covered three types of piezofoils:

- $28 \mu \mathrm{m}$ thick of the laminated foil;

$-28 \mu \mathrm{m}$ and $52 \mu \mathrm{m}$ thick of foils without laminate.

The tests concern pressure changes sensitivity and a comparison obtained results with the results obtained by means of the reference fiber sensor. Exemplary simultaneous readings from both sensors are shown in Fig. 5 while the pressure was being changed.

A direct comparison of the results obtained with a piezofoil and fiber sensor is quoted (Fig. 6). The relation between data from both sensors is not linear. There is a slight phase shift of unknown origin (Fig. 6a). After compensation of the phase difference, the relation is almost linear (Fig. 6b). Two causes of this effect were considered. The first of them is the time difference between data acquisition from both sensors, and the second 


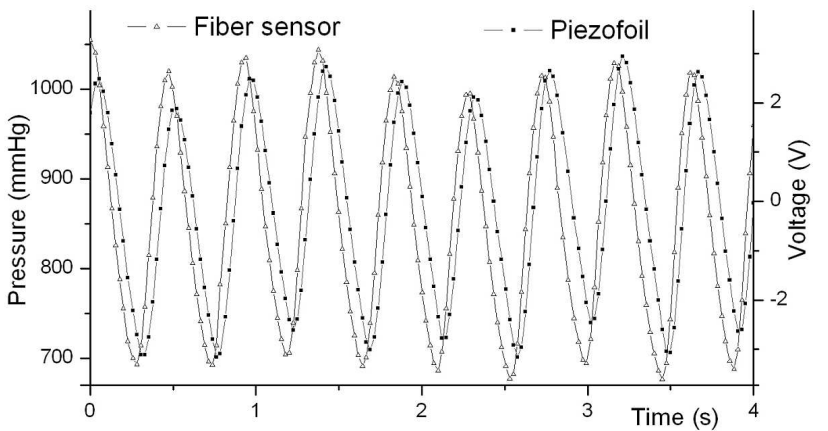

Fig. 5. Measurement results of the piezofoil test.

one was the piezofoil inertness. Neither of these causes could be definitely ruled out. Even after compensation the reproducibility of results was very low. There were differences between results from foils of the same type.

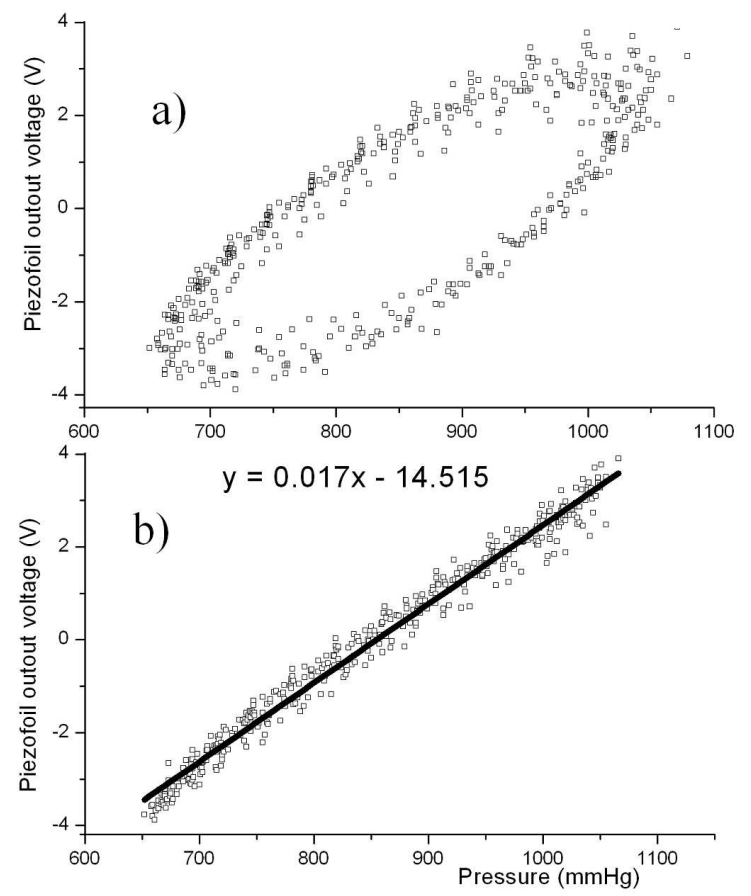

Fig. 6. Piezofoil output signal versus pressure inside cavity with uncompensated (a) and compensated (b) phase shift.

The results shown in Fig. 6 were ones of the best acquired of all carried out measurements. The sensors were very sensitive to temperature and the movement of the sensing element and other factors. Their base voltage was floating - sensor readings of the same induced pressure, during long term measurements, varied significantly.

Since while working with POLVAD prosthesis, there is limited control of environment variables (temperature, light intensity, movement of prosthesis) that could influence the piezofoils readouts, they were proven inadequate for use in this certain application.
Artificial heart project requires a high accuracy, stability and safety of measurements - this kind of requirements could not be met with the tested sensors.

\section{Polyurethane membrane tests}

An important project demand was to find a way to separate the pressure sensor from the blood environment. The proposed solution to this problem was blood pressure measurement through prosthesis wall. In the final project POLVAD its walls ought to be made in certain points thin enough, to transmit the inner pressure changes outside. To find out if this would be possible, polyurethane foils, made of the same materials as prosthesis, of different thickness were tested. For these purposes a special testing element has been made (Fig. 7).

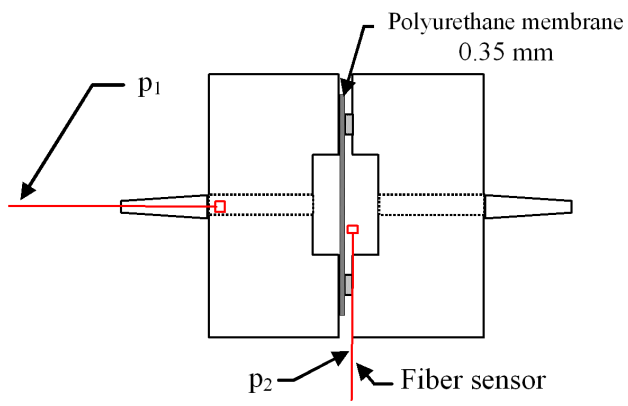

Fig. 7. Arrangement of sensors in testing chamber.
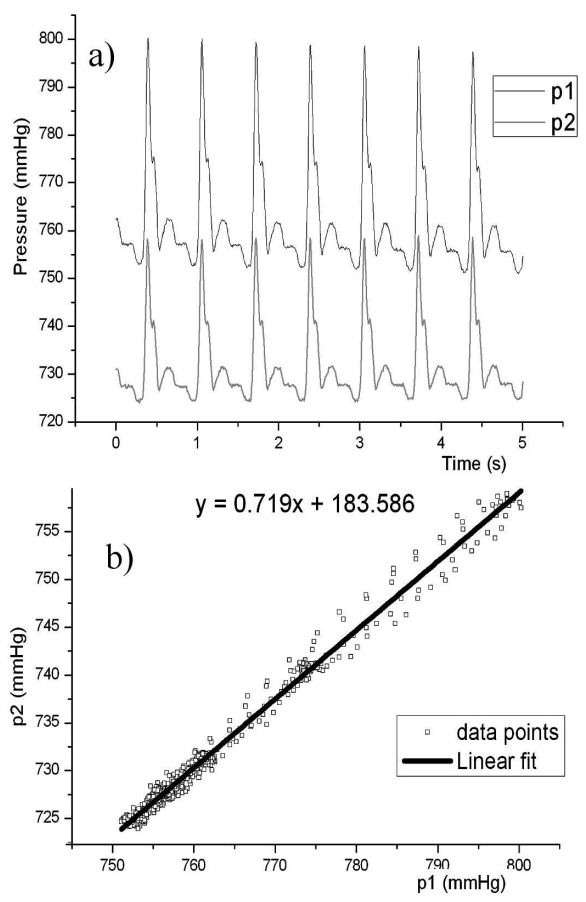

Fig. 8. Signal on both sides of the membrane (a) and relation between the sensors (b). 
The testing chamber was divided into two cavities by thin polyurethane foils. The chamber volume was filled with water and the FOP-M sensors were situated on both sides of the membrane (Fig. 7). The induced pressure was measured on " $p_{1}$ " sensor side, and the transmitted pressure was measured on the other side of the membrane by a " $p_{2}$ " sensor.

Since the pressure transmission highly depends on the foil thickness, the test has been conducted on the thickest one $(0.35 \mathrm{~mm})$ supplied with Foundation of Cardiac Surgery Development, where the measurement was made simultaneously in both chambers (Fig. 8a). Linearity between the results acquired from both sensors can be observed (Fig. 8b). The properties of the foils have a significant influence on the result but in this case, the main cause of spread of results is the presence of air bubbles inside the testing chamber, which was really difficult to be avoided. Nevertheless, the conducted research indicates that transmission of pressure through, a sufficiently thin, polyurethane membrane is possible and promising for use in the final sensor system design.

\section{Conclusions}

The main goal of the conducted research was to choose and test pressure sensors for future incorporating in POLVAD prosthesis. It is the first part of a project aiming at the introduction of a fully functional, noninvasive blood pressure measurement system, measuring pressure in the required range $(-100-400 \mathrm{mmHg})$ with a dynamicity of $5 \mathrm{mmHg} / \mathrm{ms}$, for using in the Polish Artificial Heart.
The conducted researches bring a new solid base for a future measurement system. During the studies piezoelectric foils have proved to be inappropriate for this project. The fiber pressure sensor has been chosen for future investigations. The noninvasiveness of blood pressure measurements will be obtained by the VAD casing at certain points on the prosthesis, where polyurethane material will be made thin enough to transmit changes of the blood pressure to the external pressure sensor. Future development plans include designing of measurement points and tests on the VAD prosthesis model.

\section{References}

[1] Z. Religa, R. Kustosz, Mechanical Heart Supporting. Coronary Vessels Surgery, PZWL, Warsaw 2002, p. 158 (in Polish).

[2] M. Darlak, Z. Opilski, M. Gawlikowski, R. Kustosz, T. Pustelny, Artificial Organs 31, A50 (2007).

[3] T. Pustelny, P. Struk, Z. Nawrat, M. Gawlikowski, Europ. Phys. J. - Special Topics 154, 165 (2008).

[4] M. Gawlikowski, T. Pustelny, B. Przywara-Chowaniec, P. Struk, Acta Phys Pol. 114, A-79 (2008).

[5] M. Darlak, T. Pustelny, M. Gawlikowski, R. Kustosz, Mol. Quant. Acoust. 27, 89 (2006).

[6] T. Pustelny, P. Struk, Z. Nawrót, M. Gawlikowski, Europ. Phys. J. — Special Topics 154, 161 (2008).

[7] http://www.frk.pl/frk/ .

[8] FISO - Fiber Optical Pressure Sensors http://www.fiso.com/modules/ . 